\title{
RENDIMIENTO ACADÉMICO DE ESTUDIANTES DE DISTINTO NIVEL SOCIOECONÓMICO EN UNIVERSIDADES: EL CASO DE LA PONTIFICIA UNIVERSIDAD CATÓLICA DE CHILE
}

\section{Introducción}

Existe un creciente interés por la equidad en la educación superior en Chile. Este interés refiere no solo al acceso, sino que también a los resultados de los alumnos en este nivel educativo, principalmente en relación con el rendimiento académico. Estos resultados son relevantes, ya que en el ámbito de las políticas públicas para la educación superior se espera no solo que los estudiantes ingresen al sistema, sino que también puedan finalizar sus estudios de manera exitosa (Microdatos, 2008). Pese a esta creciente atención, los estudios que indagan en los resultados académicos de los alumnos en la educación superior, y en los factores que influyen en ellos, son todavía escasos en nuestro país.

Este estudio tiene como foco conocer si existen diferencias relevantes en el desempeño académico (notas y persistencia) de jóvenes de distinto nivel socioeconómico al interior de la UC. Conocer el desempeño en la educación superior de estudiantes de distinto origen social resulta relevante para informar las políticas relacionadas con la admisión a la educación superior, financiamiento y apoyo estudiantil, y diseño e implementación curricular, tanto a nivel nacional como institucional. Asimismo, estudiar el desempeño de los distintos grupos socioeconómicos en la UC entrega antecedentes en relación con la forma en que jóvenes de distintos contextos sociales responden a los desafíos académicos de la vida universitaria en una institución con altos estándares académicos. Se trata de un aspecto esencial en el caso de la UC, una universidad selectiva y compleja, con poca representación de jóvenes de menor nivel socioeconómico, pero con un creciente interés por su inclusión en sus aulas. 
Para esta investigación se utilizaron las cohortes de ingreso 2007-2010, ya que al momento de la investigación eran estos los años para los que la UC disponía de información socioeconómica de sus alumnos entregada por el Ministerio de Educación (Mineduc). Este periodo coincide con una importante expansión de las oportunidades de financiamiento estudiantil disponibles desde el Mineduc, así como también con los nuevos mecanismos de admisión a las universidades del Consejo de Rectores de las Universidades Chilenas (CRUCH), que ponen énfasis en el desempeño escolar de los postulantes (cupos supernumerarios $^{4}$ de la Beca de Excelencia Académica).

En cuanto a la organización de este documento, primero se presentan antecedentes del sistema de educación superior en Chile, profundizando en el acceso a este nivel educativo y en los factores sociodemográficos relevantes a la hora de predecir los resultados académicos según la literatura nacional e internacional. Posteriormente, se presentan las preguntas de investigación y la metodología. Luego, se muestran los resultados organizados en dos secciones: en la primera se analiza el promedio ponderado acumulado según dependencia educativa del colegio de egreso y quintil de ingreso familiar; en tanto que en la segunda sección se presenta la persistencia para los mismos grupos definidos anteriormente. Se finaliza con una discusión en la cual se retoman los principales hallazgos de la investigación y se analizan dichos resultados a la luz de la literatura en torno al tema, incluyéndose además las limitaciones y proyecciones de la investigación.

\section{Antecedentes}

En Chile se distinguen principalmente cuatro tipos de instituciones de educación superior: universidades del CRUCH, universidades privadas sin aporte fiscal (creadas con posterioridad a 1981), institutos profesionales (IP) y centros de formación técnica (CFT). Las universidades pertenecientes al CRUCH cuentan con un sistema unificado de admisión administrado por el Departamento

\footnotetext{
4 Las universidades del CRUCH y las universidades adscritas al sistema ofrecen cupos supernumerarios (cupos adicionales a las vacantes ofrecidas de manera regular) a aquellos postulantes que obtienen Beca de Excelencia Académica (BEA) y que, habiendo postulado a estas universidades, se ubican en las listas de espera de cada carrera.
} 
de Evaluación, Medición y Registro Educacional (DEMRE) ${ }^{5}$, cuyos principales indicadores de admisión son la Prueba de Selección Universitaria (PSU) y las notas obtenidas por los alumnos en la enseñanza secundaria.

Pese a que se ha registrado un constante aumento en la cobertura de la educación superior en Chile en las últimas décadas, siguen existiendo importantes diferencias por nivel socioeconómico en el acceso a este nivel educativo: según la Encuesta CASEN 2011, la tasa neta de cobertura en educación superior en el quintil de mayores ingresos es de 59\%, mientras que en el primer quintil esta cifra es de 22\% (Ministerio de Desarrollo Social, 2013). Esta diferencia cobra mayor importancia cuando además se considera la matrícula de alumnos de distinto nivel socioeconómico por tipo de institución. Según destaca un informe de la Organisation for Economic Co-operation and Development (OECD), en Chile existen marcadas diferencias en el tipo de institución al que acceden los jóvenes según su nivel socioeconómico, siendo los estudiantes de menores recursos mucho más proclives a matricularse en instituciones técnicas (OECD, 2009 , p. 83), las que en su mayoría no están acreditadas (Servicio de Información de Educación Superior, 2013). Un estudio de Castillo y Cabezas (2010) que caracterizó a los jóvenes de "primera generación", es decir, con padres sin estudios terciarios, mostró resultados similares al evidenciar diferencias en el tipo de institución en la que se matriculan estos estudiantes y al compararlos con sus pares de padres que sí alcanzaron la educación terciaria.

La menor participación de los jóvenes de menor nivel socioeconómico en universidades está relacionada en gran medida con el proceso de selección de estudiantes que realizan estas instituciones, basado principalmente en los puntajes PSU, lo que limita las posibilidades de jóvenes de menores recursos para acceder a estos establecimientos, al obtener sistemáticamente menores puntajes en dicha prueba (OECD, 2009). Esta situación podría tener consecuencias tanto en la experiencia educativa de estos jóvenes, como en su posterior inserción el ámbito laboral.

Desde el proceso de admisión 2012, ocho universidades privadas no tradicionales participan de este sistema unificado de admisión para seleccionar a sus estudiantes. 
En el caso de la UC, las cifras de la Dirección de Servicios y Registros Docentes (DSRD) y de la Dirección de Asistencia Socioeconómica (DASE), analizadas por las autoras, revelan que durante el periodo 2007-2011 la mayoría de los alumnos matriculados en la UC pertenecía al quintil de mayor ingreso (quinto quintil), representando a más de un $70 \%$ del total de alumnos, mientras que la participación de estudiantes del primer y segundo quintil fue considerablemente menor, cada uno con cifras por debajo del 10\% de la matrícula total (ver Tabla 1 en el Anexo 1)6. Asimismo, la proporción de estudiantes proveniente de colegios particulares pagados alcanzó cifras superiores a los dos tercios del total de matriculados por el proceso de admisión basado en el puntaje PSU (admisión ordinaria o regular) en el periodo 1999-2011. En tanto, si bien la participación de los alumnos de establecimientos que reciben subsidios del Estado se mantuvo relativamente constante en igual periodo, la participación en la matrícula de los alumnos provenientes de colegios subsidiados de administración municipal disminuyó de aproximadamente un 19\% a un 13\% (ver Figura 1 en el Anexo 1).

Para los jóvenes provenientes de entornos vulnerables, acceder a la educación superior presenta grandes desafíos y el grado de inserción que logren en este nivel educacional, tanto académico como social, dependerá no solo de sus características particulares, sino que de las características de la institución en la que se matriculan (Leyton, Vásquez y Fuenzalida, 2012). Por su parte, para las instituciones de educación superior, en especial para aquellas con mayores estándares académicos, recibir a estudiantes de menor nivel socioeconómico significa un reto, al existir la impresión de que estos jóvenes, incluso si logran ser admitidos, se encuentran menos preparados académicamente para beneficiarse de la experiencia universitaria, requiriendo por lo tanto mayor apoyo para la nivelación académica (OECD, 2009). En la próxima sección se revisará lo que ha dicho la literatura nacional e internacional respecto de las diferencias en el

6 Existe una considerable relación entre el quintil de ingreso familiar y la dependencia educativa del colegio de egreso del estudiante en el periodo analizado: en la UC, entre quienes estudiaron la enseñanza media en un colegio particular pagado, más del 90\% pertenecía al quinto quintil, en tanto que para los alumnos que estudiaron en colegios municipales, esta cifra bordeó apenas el 20\% (ver Tabla 2 en el Anexo 1). 
desempeño académico universitario entre grupos de mayor y menor nivel socioeconómico.

\subsection{Resultados académicos en la educación superior ${ }^{7}$}

Tanto la literatura nacional como internacional señala que el desempeño académico del estudiante antes de ingresar a una institución de educación superior es el mejor predictor de su rendimiento académico en este nivel educativo, en particular las notas obtenidas en la educación secundaria y, en segundo lugar, los resultados en pruebas estandarizadas de selección (Geiser y Studley, 2002; Manzi, Bravo, Del Pino, Donoso, Martínez y Pizarro, 2006). En esta misma línea, algunas investigaciones nacionales han mostrado la relevancia del ranking de notas de enseñanza media en la predicción del desempeño académico en la educación superior, tanto a nivel de sistema (Contreras, Gallegos y Meneses, 2009) como de programas específicos (Meneses y Toro, 2012; Koljatic y Silva, 2012; Gil y Del Canto, 2012).

Por limitaciones en la extensión del artículo y debido al foco del estudio en aspectos socioeconómicos, en esta sección se ahonda en estudios nacionales e internacionales que han explorado las variables sociodemográficas y de financiamiento asociadas a las notas y persistencia en la educación superior. Sin embargo, a la hora de discutir los resultados de la presente investigación, es necesario tener en cuenta la importante relación entre los indicadores académicos anteriores y los resultados en la educación superior, dadas las exigencias que hay en la admisión a la UC en términos de excelencia académica en los estudios secundarios.

En esta sección se presenta en primer lugar la evidencia relacionada con la persistencia y luego aquella referida a las notas en la educación superior. Cabe destacar que esta revisión bibliográfica

Existen distintos tipos de resultados en educación superior y pueden desglosarse en tres dimensiones principales: cognitiva, interpersonal e intrapersonal (Oswald, Schmitt, Kim, Ramsay \& Gillespie, 2004). Dentro de la dimensión cognitiva se encuentran los indicadores académicos considerados en este estudio, como las calificaciones obtenidas por los estudiantes, los porcentajes de aprobación de asignaturas, la retención o deserción, las tasas de graduación, etc. 
está enfocada en investigaciones empíricas que permitan comparar los resultados de este estudio, sin desconocer la importancia de la literatura que ha abordado las diferencias académicas entre distintos grupos socioeconómicos en el contexto de la construcción de modelos teóricos que expliquen dichas diferencias (Bourdieu y Passeron, 2001; Bernstein, 1989).

\subsection{Persistencia en la educación superior}

Los estudios que han examinado el desempeño académico en la educación superior en Chile han indagado principalmente en los niveles de persistencia obtenidos, debido a la mayor comparabilidad de este indicador entre instituciones y carreras, y a su carácter sustantivo en términos de continuación de estudios terciarios. Las cifras entregadas en estas investigaciones indican que la retención hasta segundo año, en la misma institución, para las cohortes de ingreso 2007, 2008 y 2009 es cercana al 70\%, con importantes diferencias según el tipo de institución. Lideran la lista las universidades del CRUCH de administración privada, entre las que se incluye la UC, con tasas que superan el 80\% para las cohortes 2007 y 2008 (Servicio de Información de Educación Superior 2008, 2009 y 2010).

En el marco de un estudio que buscaba indagar en los factores que influyen en la deserción en la educación superior, la investigación de Santelices, Catalán, Horn y Kruger (2013) encontró que la persistencia hasta el segundo año en la misma institución es de aproximadamente un 74\%, decreciendo a través de los años para alcanzar una cifra de alrededor de un 53\% en la retención hasta quinto año. Este estudio también muestra importantes diferencias en las tasas de persistencia al interior de los distintos tipos de institución, siendo las universidades del CRUCH las que alcanzan los mayores niveles de persistencia, en tanto que en las instituciones técnicas estos porcentajes son notablemente menores.

Las investigaciones acerca de la persistencia en Chile se han desarrollado principalmente con el objetivo de dimensionar el aporte de la ayuda financiera en la continuación de estudios superiores, controlando a través de diversas variables. Las variables sociodemográficas más estudiadas han sido el nivel educacional de los 
padres y la dependencia del establecimiento al que asistió el alumno durante su enseñanza media. Al estudiar la relación entre financiamiento estudiantil y persistencia, controlando mediante otras variables, estudios nacionales (Barrios, Meneses y Paredes, 2011; Centro de Estudios Mineduc, 2012) son concluyentes en encontrar mayor probabilidad de desertar en alumnos cuyos padres no habían completado la enseñanza secundaria en relación con aquellos cuyos padres contaban con estudios universitarios completos. Barrios et. al. (2011) encontraron que los alumnos con padres que no habían completado la enseñanza media tenían una mayor probabilidad de desertar al tercer año comparados con los alumnos cuyos padres contaban con estudios universitarios completos. El efecto de esta variable se mantiene, pero la significancia disminuye al controlar el tipo de colegio de enseñanza media al que asistió el estudiante. En la misma línea, la investigación del Centro de Estudios del Mineduc (2012) encontró que la probabilidad de persistir a segundo año en el sistema de educación superior es significativamente menor en alumnos cuyos padres no completaron la enseñanza secundaria en relación con aquellos con padres universitarios, incluso controlando otras variables de nivel socioeconómico, como ingreso familiar y tipo de establecimiento de enseñanza media. Es interesante notar que los coeficientes asociados a las otras categorías de nivel educativo (educación media completa y educación superior incompleta) no fueron significativos.

$\mathrm{Al}$ igual que en Chile, muchas de las investigaciones que han abordado los determinantes de la deserción en la educación superior en Estados Unidos, lo han hecho en el marco de investigar el rol de la ayuda financiera en la persistencia, controlando otras variables sociodemográficas. Dichos estudios han explorado frecuentemente las diferencias por nivel socioeconómico. Mientras Kuh, Kinzie, Buckley, Bridges y Hayek (2006) reportaron que los jóvenes con padres que no cuentan con educación superior muestran mayores probabilidades de desertar, Dynarski (2004) encontró que los jóvenes de familias con bajos ingresos tienen, por un lado, menores probabilidades de acceder a la educación superior, y por otro, una mayor probabilidad de desertar una vez que ingresan a ella. Como destaca Tinto (2004), esto estaría relacionado tanto con factores culturales y económicos, como con aspectos de preparación académica. Al respecto, St. John, 
Hu y Fisher (2011) señalan que el menor conocimiento que tienen los jóvenes de bajo nivel socioeconómico respecto del sistema educativo podría afectar negativamente su persistencia.

Es importante destacar, sin embargo, que al igual que otros resultados académicos como las notas o las tasas de graduación, la persistencia podría estar influida por factores (académicos y no académicos) posteriores a la admisión, tales como aspectos motivacionales y vocacionales (Microdatos, 2008; Canales y De los Ríos, 2007; González, Uribe y González, 2005) y académicos, como las notas en el primer año de la enseñanza superior (Pascarella y Terenzini, 2005; Allen, Robbins, Casillas y Oh, 2008). Asimismo, si bien la literatura constata una asociación entre el nivel socioeconómico y persistencia en la educación superior, la magnitud de ese efecto para alumnos de una misma institución es moderada o pequeña (Geiser y Santelices, 2007).

\subsection{Notas en la educación superior}

En Chile, las variables sociodemográficas más utilizadas en la predicción de rendimiento académico en la educación superior refieren al nivel socioeconómico de las familias. Los estudios de Manzi (2006) y Santelices (2007) indican que el nivel educativo de los padres del estudiante pareciera no presentar una relación importante con su rendimiento académico en la educación superior. Si bien Santelices (2007) reportó una relación estadísticamente significativa, esta no presenta un tamaño importante a la hora de explicar el promedio ponderado acumulado de los estudiantes (coeficientes menores a $0,1)$. Es importante notar, sin embargo, que en ambos estudios los resultados refieren a grupos de estudiantes que lograron ingresar a universidades que participan del proceso de admisión centralizado del CRUCH, superando por lo tanto la barrera de entrada de los puntajes PSU exigidos por estas instituciones.

A nivel internacional y, especialmente en Estados Unidos, existe una amplia literatura acerca de los factores académicos y no académicos que permitirían predecir las calificaciones en la educación superior. Estas investigaciones llegan a resultados similares respecto de la escasa importancia de las variables sociodemográficas a la hora 
de explicar el rendimiento universitario, una vez que se consideran las notas de educación secundaria y el puntaje en pruebas estandarizadas (Geiser y Studley, 2002; Zwick, 2002; Geiser y Santelices, 2006). Sin embargo, Rothstein (2004) sostiene que el pequeño efecto marginal del nivel socioeconómico en este tipo de estudios se debería a que los resultados en las pruebas estandarizadas de admisión a la universidad absorben de manera considerable el contexto social de los estudiantes, por lo que el efecto predictivo de variables de nivel socioeconómico sobre los resultados en la educación superior estaría subestimado en modelos que consideran también el puntaje en pruebas estandarizadas.

En Chile, hasta donde se tiene conocimiento, no hay estudios enfocados puntualmente en analizar las diferencias en el desempeño académico de alumnos de distinto nivel socioeconómico al interior de instituciones específicas de educación superior. Si bien se sabe que las tasas de persistencia, y presumiblemente también las de graduación, de los alumnos en instituciones del CRUCH son mayores que las del resto de las instituciones de educación superior (SIES, 2008; 2009; Santelices et al., 2013) se desconocen las diferencias en el desempeño académico entre distintos grupos al interior de dichas instituciones. La evidencia internacional sugiere que las diferencias entre grupos socioeconómicos tenderían a aminorarse al considerar estudiantes de una misma institución (Geiser y Santelices, 2007).

En el marco de estas interrogantes se espera que esta investigación aporte al conocimiento del desempeño académico de jóvenes de distintos grupos socioeconómicos al interior de una misma institución de educación superior. Esta es una pregunta que resulta relevante frente al aumento de iniciativas de admisión y financiamiento que buscan aumentar la diversidad social en las instituciones de educación superior. Es importante conocer cómo los jóvenes de distinto origen social enfrentan las exigencias académicas y en qué medida son capaces de persistir en sus estudios, en especial en instituciones de alta exigencia, como la UC. Más ampliamente, se trata de una pregunta de interés para todas las universidades, tomando en cuenta la cada vez mayor participación de jóvenes de menor nivel socioeconómico en el sistema. 


\section{Preguntas de investigación}

Este estudio intenta responder las siguientes preguntas:

- ¿Existen diferencias en las notas universitarias obtenidas por alumnos matriculados en la UC provenientes de distintos tipos de establecimientos educativos (particulares pagados, particulares subvencionadosymunicipalizados)y de distintosquintiles de ingreso?

- ¿Existen diferencias en las tasas de persistencia observadas en la UC entre alumnos de estos distintos grupos?

\section{Metodología}

\subsection{Fuentes de información}

La información necesaria para esta investigación fue entregada por la Dirección de Servicios y Registros Docentes (DSRD) y de la Dirección de Asistencia Socioeconómica (DASE) de la UC.

\subsection{Muestra}

La muestra corresponde a las cohortes de ingreso 2007, 2008, 2009 y 2010 que ingresaron a la UC vía admisión ordinaria (mediante la PSU, lo que corresponde a entre 3.500 y 3.800 alumnos por cada cohorte de ingreso en el periodo estudiado) ${ }^{8}$. DASE cuenta con información socioeconómica de sus alumnos a partir del año 2007, la cual ha sido proporcionada tanto por el Mineduc, como a partir de informes de acreditación socioeconómica propios, que son los que se ocuparán en este análisis. De este modo, para mantener la consistencia de la información, el análisis acerca de la dependencia educacional, promedio ponderado acumulado y persistencia se realizará para las cohortes mencionadas ${ }^{9}$.

8 Para mayores detalles del rendimiento diferencial entre alumnos de admisión ordinaria y especial, ver Anexo 2.

9 Al momento de realizar esta investigación no se disponía aún de información de rendimiento académico para la cohorte 2011. Estas cohortes son anteriores a la implementación del programa de Talento e Inclusión UC, el que podría haber modificado el cuerpo de estudiantes que ingresan a la universidad. 


\subsection{Variables}

Se seleccionó un conjunto de variables que permitiera dar una aproximación a las dos dimensiones de interés, en particular, nivel socioeconómico y rendimiento académico universitario. Estas variables se seleccionaron tanto por su disponibilidad para las cohortes estudiadas, como por su relevancia en la experiencia universitaria y uso en investigaciones similares.

\section{Nivel socioeconómico}

- Dependencia educativa del colegio de egreso: municipal, particular subvencionada (ambos con subvención estatal) y particular pagada.

- Quintil de ingreso familiar: para esta investigación se usó el quintil de ingreso calculado por DASE, el que, además del ingreso reportado por el grupo familiar, incluye propiedades y vehículos, contemplando en su cálculo eventuales gastos que afecten de manera importante el presupuesto de cada familia. En general estos quintiles son homologables a aquellos reportados por el Mineduc, aunque existen algunas diferencias, debido a que la institución gubernamental solo incluye el ingreso familiar en sus cálculos ${ }^{10}$.

\section{Rendimiento académico}

- Promedio ponderado acumulado (PPA): indicador de rendimiento académico que incorpora todas las notas obtenidas por el estudiante hasta un determinado periodo académico, ponderadas según su respectiva cantidad de créditos. Cabe destacar que si bien el promedio es una medida que presenta sensibilidad a valores extremos, su uso en esta investigación es pertinente al ser uno de los indicadores más utilizados en la literatura para medir el desempeño académico.

- Persistencia institucional: esta variable se refiere a la persistencia dentro de la institución, pudiendo haber cambio de carrera, y

10 Es importante destacar que no todos los estudiantes realizan la acreditación socioeconómica en la universidad, y debido a que esta es imprescindible para postular a beneficios socioeconómicos internos, es posible asumir que aquellos que no realizan esta evaluación pertenecen al quintil de mayores ingresos. 
fue evaluada en distintos periodos académicos. Esta fue creada a partir de la variable "situación académica" de los alumnos en la UC, reportada por la DSRD. Para determinar si el estudiante persiste en la institución en el periodo académico en cuestión, se recodificaron los valores "regular" y "ausente" (por suspensión, por suspensión de salud, por fuerza mayor, por sanción académica, por anulación, por no inscripción en curso, etc.) indicando persistencia, y el valor "no vigente" (abandono, renunciado, etc.) para indicar la no persistencia o deserción. En el caso de la no vigencia por egreso o titulación, el estudiante quedó calificado como "egresado". Es importante destacar que se considera a los ausentes como "persistentes", ya que estos alumnos podrían eventualmente retomar sus estudios sin tener que ser readmitidos por la universidad. Este no es el caso de los alumnos no vigentes, quienes deben serlo en caso de querer reanudar sus estudios.

\subsection{Análisis}

Se realizó un análisis descriptivo de carácter exploratorio para indagar en las relaciones entre el nivel socioeconómico y el desempeño académico. Se utilizaron tablas de contingencia con las variables de interés y las diferencias entre grupos se analizaron utilizando pruebas ANOVA de un factor con comparaciones múltiples post hoc para los análisis de promedio ponderado acumulado según dependencia y quintil, al contar con más de dos grupos de comparación; en particular se utilizó el Test de Bonferroni, cuyos resultados se reportan en las tablas de manera resumida ${ }^{11}$. De modo de evaluar el cumplimiento del supuesto de igualdad de varianzas que exige esta prueba, se corrieron Test de Levene en conjunto con las pruebas ANOVA; estos fueron reportados en las tablas solo en los casos en los que no se cumpliera la igualdad de varianza (significancia menor a 0,05). El análisis de las diferencias en persistencia, al tratarse de proporciones, se realizó con la prueba de Chi-Cuadrado que se complementó con la prueba $Z$ de comparación entre columnas, al existir más de dos grupos de comparación.

11 Se indican con un * las diferencias estadísticamente significativas a un 95\% de confianza, explicando entre qué grupos y a favor de qué grupo se dan dichas diferencias. 
34 RENDIMIENTO ACADÉMICO DE ESTUDIANTES DE DISTINTO NIVEL SOCIOECONÓMICO EN UNIVERSIDADES: EL CASO DE LA PONTIFICIA UNIVERSIDAD CATÓLICA DE CHILE - X. Catalán y M. V. Santelices

\section{Resultados}

A continuación se presentan los principales resultados obtenidos en la investigación.

\subsection{Promedio ponderado acumulado}

\subsubsection{Rendimiento académico según dependencia educacional}

Al indagar en el PPA de los alumnos de las cohortes 2007-2010 (Tabla 1) no se observaron diferencias con magnitudes importantes al segmentar por dependencia educativa del colegio de egreso. Los alumnos provenientes de colegios particulares pagados obtuvieron en promedio calificaciones levemente superiores (entre una y dos décimas más) que sus pares de colegios municipales y particulares subvencionados en todos los periodos, bordeando el promedio 5,0 en el primer semestre y aumentando levemente en los periodos siguientes. Estas diferencias entre alumnos de distinto tipo de establecimiento, si bien son estadísticamente significativas para la mayoría de los periodos analizados (Test ANOVA con un 95\% de confianza), no son de magnitud importante en lo práctico ${ }^{12}$.

Por otra parte, las diferencias entre los alumnos de colegios particulares subvencionados y municipales no son estadísticamente significativas. Los alumnos provenientes de estos dos tipos de establecimientos presentan promedios en torno al 4,8 durante el primer semestre y aumentan relativamente más sus promedios a medida que avanzan en sus estudios que quienes provienen de colegios particulares pagados, acercándose a los PPA de este último grupo a medida que transcurren los semestres. Cabe destacar que este incremento en el tiempo probablemente está relacionado con la renuncia de alumnos de bajo rendimiento.

12 Si bien en Chile las calificaciones generalmente se expresan con una décima, en este análisis se presentan resultados con dos decimales para hacer más evidentes las diferencias entre grupos. La calificación 4,0 es el nivel mínimo de aprobación de cursos en Chile. 


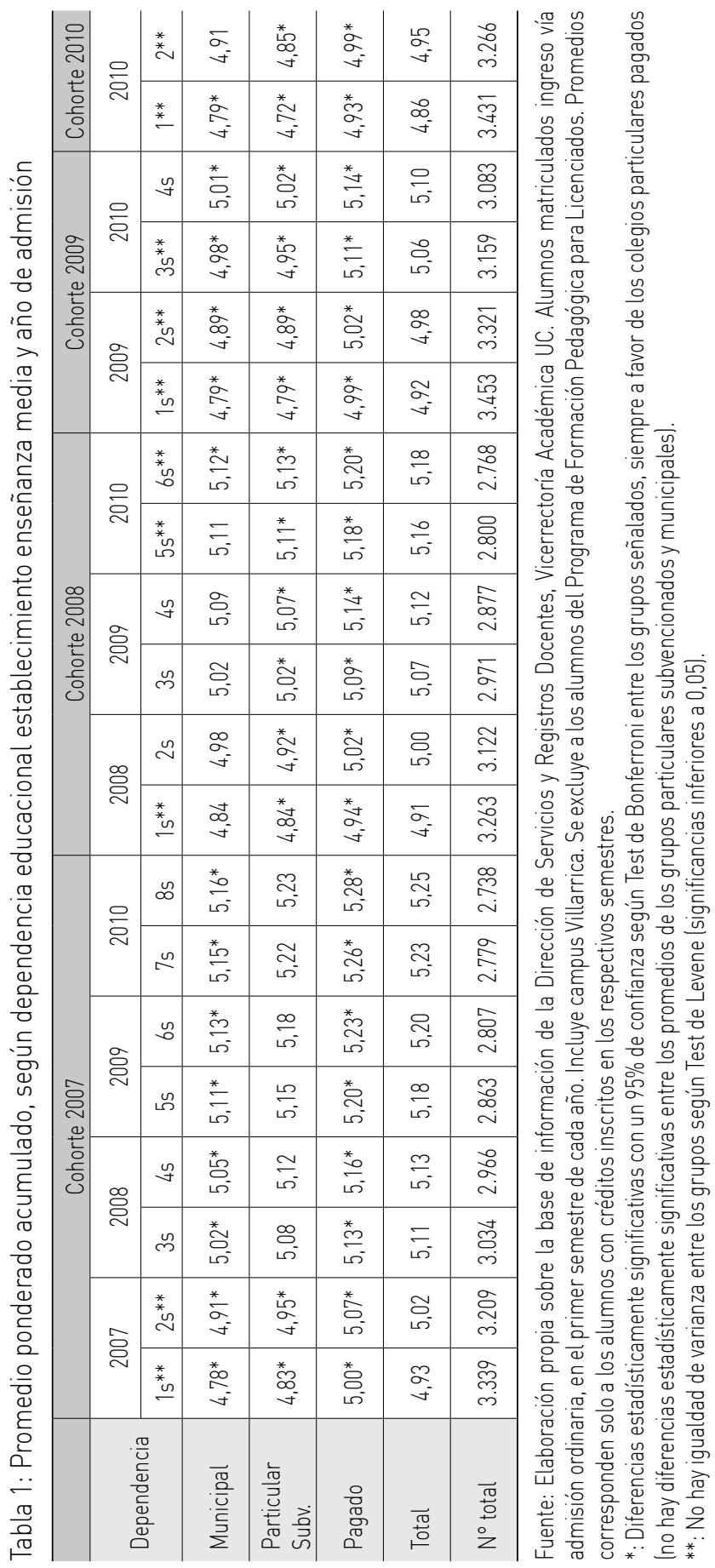




\subsubsection{Rendimiento académico según quintil de ingreso}

Al igual que para el análisis hecho para dependencia, hay dos conclusiones importantes del análisis por quintil de ingreso socioeconómico de los estudiantes: los promedios ponderados acumulados mejoran en el tiempo para todas las cohortes y para todos los quintiles, y si bien algunas de las diferencias entre grupos son estadísticamente significativas, estas son de escasa importancia práctica. En particular, la prueba ANOVA y de comparaciones post hoc arrojó diferencias estadísticamente significativas entre grupos, con un 95\% de confianza, a favor del quinto quintil versus los quintiles dos y cuatro, para la mayoría de los semestres analizados.

Pese a este desempeño relativamente similar entre los distintos grupos, para las cuatro cohortes de admisión analizados, los alumnos del quinto quintil son los que presentan mejores calificaciones promedio (cercanas al promedio 5,0) en todos los semestres, mientras que, en general, el segundo quintil es el que obtiene los promedios más bajos (entre una y dos décimas menos que el quinto quintil). Para mayores detalles ver la Tabla 2. 


\begin{tabular}{|c|c|c|c|c|c|c|c|c|c|c|}
\hline 으 & \multirow{2}{*}{ 를 } & $\stackrel{*}{\stackrel{*}{\sim}}$ & \begin{tabular}{l}
$*$ \\
0 \\
0 \\
\hdashline \\
\hdashline
\end{tabular} & \begin{tabular}{l}
$*$ \\
* \\
$\vdots$ \\
\hdashline \\
$\forall$
\end{tabular} & $\begin{array}{l}\mathscr{D} \\
\mathscr{O} \\
\text { - }\end{array}$ & $\underset{\sigma}{q}$ & $\begin{array}{l}* \\
0 \\
o \\
\forall\end{array}$ & 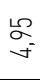 & 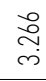 & \multirow{2}{*}{ 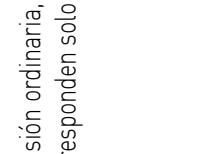 } \\
\hline స్ర & & $\stackrel{*}{*}$ & $\begin{array}{l}* \\
0 \\
0 \\
\sigma^{-}\end{array}$ & 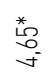 & $\stackrel{*}{\stackrel{*}{\gtrless}}$ & $\begin{array}{l}\text { OD } \\
\text { - }\end{array}$ & \begin{tabular}{l}
$\stackrel{*}{\alpha}$ \\
\multirow{\sigma}{*}{}
\end{tabular} & $\begin{array}{l}\infty \\
\infty \\
\sigma^{-}\end{array}$ & 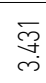 & \\
\hline \multirow{4}{*}{ ర్లి } & \multirow{2}{*}{ 음 } & \& & $\underset{8}{\stackrel{9}{\sigma}}$ & 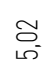 & $\underset{\gamma}{\alpha}$ & $\begin{array}{l}\text { Oे } \\
\sigma^{\circ}\end{array}$ & $\frac{7}{E}$ & $\bar{E}$ & ஜू & 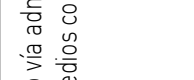 \\
\hline & & $\stackrel{m}{m}$ & 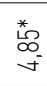 & \begin{tabular}{l}
$\stackrel{*}{\alpha}$ \\
\multirow{\sigma}{*}{} \\
\end{tabular} & $\begin{array}{l}* \\
\stackrel{*}{\infty} \\
\sigma^{-}\end{array}$ & $\begin{array}{l}\text { 농 } \\
\text { 듀 }\end{array}$ & 葛 & 웅 & 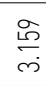 & 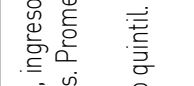 \\
\hline & ơ & $\sim$ & 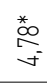 & \begin{tabular}{l}
$*$ \\
\multirow{2}{\alpha}{} \\
\multirow{2}{*}{}
\end{tabular} & 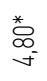 & वे & 莫 & $\stackrel{\infty}{\sigma}$ & $\underset{\widetilde{m}}{\tilde{m}}$ & $\begin{array}{l}\text { No } \frac{0}{0} \\
\frac{0}{0} . \frac{\pi}{0} \\
\frac{\pi}{3}\end{array}$ \\
\hline & $\curvearrowright$ & $\stackrel{*}{\stackrel{*}{\leftrightarrows}}$ & 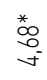 & \begin{tabular}{l}
$*_{0}^{*}$ \\
\multirow{2}{*}{}
\end{tabular} & $\begin{array}{l}\text { * } \\
0 \\
0 \\
\underbrace{-}\end{array}$ & $\underset{\sigma}{\sigma}$ & $\stackrel{\stackrel{*}{\Delta}}{\stackrel{*}{\sigma}}$ & $\underset{\sim}{\approx}$ & $\stackrel{\widetilde{R}}{\stackrel{\stackrel{\rho}{\rho}}{m}}$ & 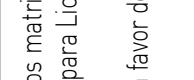 \\
\hline \multirow{6}{*}{ 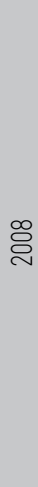 } & \multirow{2}{*}{ 응 } & ( ) & $\frac{\sigma}{5}$ & 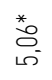 & $\frac{m}{\sigma^{\circ}}$ & $\stackrel{E}{E}$ & $\frac{*}{\circ}$ & $\frac{\infty}{5^{-}}$ & 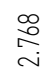 & 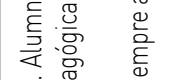 \\
\hline & & డ & $\frac{0}{50}$ & 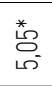 & o & $\stackrel{\circ}{5^{\circ}}$ & $\stackrel{\stackrel{*}{E}}{\sigma_{0}^{-}}$ & $\frac{0}{55^{-}}$ & $\begin{array}{l}\text { O } \\
\text { O } \\
\text { i }\end{array}$ & 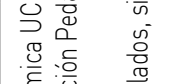 \\
\hline & 8 & f & $\underset{50}{\stackrel{\Xi}{ \pm}}$ & 䓪 & 웅 & $\frac{7}{5 \sigma^{-}}$ & $\stackrel{*}{\stackrel{*}{\sigma}}$ & $\underset{50}{\cong}$ & 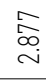 & 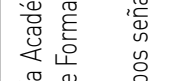 \\
\hline & & m & S్ & \begin{tabular}{l}
$\infty$ \\
\multirow{\sigma}{*}{}
\end{tabular} & $\bar{\sigma}^{-}$ & 옹 & $\begin{array}{l}\infty \\
\stackrel{\circ}{\circ} \\
\sigma^{\circ}\end{array}$ & 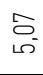 & $\stackrel{\bar{a}}{\mathrm{i}}$ & 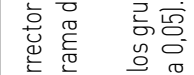 \\
\hline & o & $\stackrel{*}{*}$ & $\underset{\substack{\alpha \\
\hdashline}}{ }$ & $\underset{\sigma}{\sigma}$ & $\underset{\sigma}{\approx}$ & $\stackrel{\widehat{\sigma}}{\sigma}$ & $\underset{\widetilde{\sigma}}{\widetilde{\sigma}}$ & 焉 & $\underset{\oplus}{\stackrel{\Xi}{\Xi}}$ & 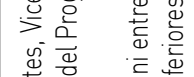 \\
\hline & $\sim$ & $\stackrel{*}{*}$ & $\begin{array}{l}\bar{\infty} \\
\dot{y}\end{array}$ & 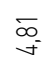 & 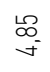 & $\underset{8}{\circ}$ & $\underset{\alpha}{\alpha}$ & $\bar{\sigma}$ & 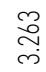 & \\
\hline & 으 & 䊝 & $\underset{\sim}{\bar{\tau}}$ & $\stackrel{*}{=}$ & $\underset{⿱ 乛}{\stackrel{\sim}{\sim}}$ & $\stackrel{*}{\circ}$ & $\begin{array}{l}* \\
\stackrel{*}{0} \\
\text { ᄃ) } \\
\end{array}$ & $\underset{\sim}{\stackrel{L}{\sim}}$ & $\stackrel{\infty}{\stackrel{一}{\sim}}$ & 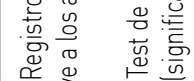 \\
\hline & $\approx$ & $\sim$ & $\frac{\sigma}{\sigma^{\circ}}$ & $\stackrel{*}{E_{5}^{-5}}$ & 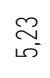 & $\stackrel{*}{\stackrel{*}{E}}$ & $\begin{array}{l}\stackrel{*}{0}^{\sim} \\
\text { డn }\end{array}$ & $\underset{\leftarrow}{\approx}$ & 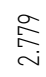 & 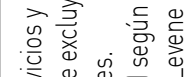 \\
\hline & o & 悉 & $\frac{0}{6}$ & 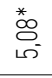 & 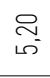 & $\stackrel{*}{\stackrel{*}{ \pm}}$ & $\stackrel{*}{\stackrel{*}{\sim}}$ & 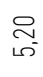 & 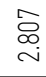 & 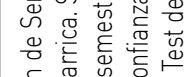 \\
\hline 5 & & డ & $\underset{5}{\cong}$ & 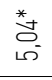 & $\underset{50}{E}$ & $\underset{\sigma^{\circ}}{\simeq}$ & $\begin{array}{l}\stackrel{*}{\sim} \\
\text { ద } \\
\end{array}$ & $\stackrel{\infty}{\sigma^{-}}$ & $\begin{array}{l}\text { ల } \\
\infty \\
\text { i }\end{array}$ & 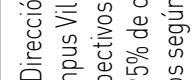 \\
\hline & $\stackrel{\circ}{\circ}$ & 䔳 & o & 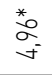 & $\frac{J}{\sigma^{-}}$ & 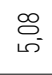 & $\stackrel{*}{\stackrel{*}{\circ}}$ & $\frac{m}{\sigma}$ & ঐ̊ & \\
\hline & & ભ & 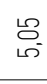 & 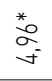 & $\underset{50}{\stackrel{O}{5}}$ & 葛 & $\stackrel{*}{\stackrel{*}{ \pm}}$ & $\bar{E}$ & 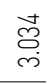 & 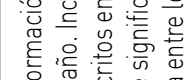 \\
\hline & 5 & $\stackrel{*}{*}$ & $\underset{\leftarrow}{\stackrel{\sigma}{\sigma}}$ & 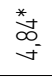 & \begin{tabular}{l}
$\infty$ \\
\hdashline \\
\multirow{j}{*}{}
\end{tabular} & 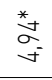 & 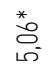 & . & 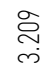 & 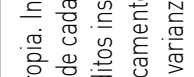 \\
\hline & & $\stackrel{*}{*}$ & $\begin{array}{l}\stackrel{L}{\infty} \\
\underset{\gamma}{+}\end{array}$ & \begin{tabular}{l}
$\underbrace{*}_{0} 0$ \\
\multirow{0}{*}{+}
\end{tabular} & $\begin{array}{l}\stackrel{L}{\infty} \\
\underset{f}{+}\end{array}$ & 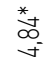 & 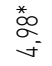 & $\forall$ & $\underset{m}{\tilde{m}}$ & 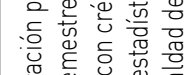 \\
\hline & 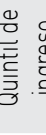 & & - & $\sim$ & $m$ & $\checkmark$ & مـ & 흐 & 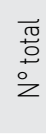 & 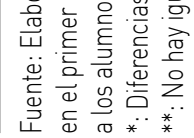 \\
\hline
\end{tabular}




\subsection{Persistencia}

4.2.1. Persistencia según dependencia educacional del establecimiento de egreso

Los niveles de persistencia en la universidad en el periodo estudiado fueron similares para los alumnos egresados de los tres grupos de dependencia educacional, con una persistencia cercana al 100\% el primer semestre, bordeando el 90\% en el tercer semestre, el $85 \%$ en el quinto semestre y el $83 \%$ en el séptimo semestre. En general, las diferencias entre tipos de dependencia administrativa del colegio de egreso no fueron estadísticamente significativas; en los periodos que sí resultaron ser significativas, lo fueron a favor de quienes egresaron de establecimientos de dependencia particular pagada (ver Tabla 3).

Tabla 3: Porcentaje de alumnos persistentes según dependencia educacional establecimiento de enseñanza media y año de admisión

\begin{tabular}{l|c|c|c|c|c|c|c|c|c|c}
\hline \multirow{2}{*}{ Dependencia } & \multicolumn{5}{|c|}{2007} & \multicolumn{4}{c|}{2008} & \multicolumn{2}{|c|}{2009} & 2010 \\
\cline { 2 - 11 } & $1 \mathrm{~s}$ & $3 \mathrm{~s}$ & $5 \mathrm{~s}$ & $7 \mathrm{~s}$ & $1 \mathrm{~s}$ & $3 \mathrm{~s}$ & $5 \mathrm{~s}$ & $1 \mathrm{~s}$ & $3 \mathrm{~s}$ & $1 \mathrm{~s}$ \\
\hline Municipal & 98,5 & 90,1 & $82,9 *$ & 80,7 & 97,8 & 89,6 & 83,4 & $96,7 *$ & 90,5 & 99,3 \\
\hline Particular Subv. & 97,9 & 90,3 & 85,2 & 81,5 & 97,2 & 89,7 & 83,8 & 98,1 & 91,0 & 97,5 \\
\hline Particular Pagado & 98,5 & 92,3 & $87,8 *$ & 84,7 & 98,2 & 91,4 & 86,3 & $98,8 *$ & 93,1 & 98,6 \\
\hline Todos & 98,3 & 91,5 & 86,5 & 83,4 & 97,9 & 90,8 & 85,3 & 98,4 & 92,3 & 98,4 \\
\hline$N^{0}$ total persiste & 3.367 & 3.133 & 2.962 & 2.855 & 3.307 & 3.067 & 2.882 & 3.500 & 3.285 & 3.463 \\
\hline
\end{tabular}

Fuente: Elaboración propia sobre la base de información de la Dirección de Servicios y Registros Docentes, Vicerrectoría Académica UC. Alumnos matriculados ingreso vía admisión ordinaria, en el primer semestre de cada año. Incluye campus Villarrica. Se excluye a los alumnos del Programa de Formación Pedagógica para Licenciados.

*: Diferencias estadísticamente significativas, con un $95 \%$ de confianza entre la dependencia particular pagada y las otras, siempre a favor de la primera. No existen diferencias estadísticamente significativas entre los alumnos de la dependencia particular subvencionada y municipal.

\subsubsection{Persistencia según quintil de ingreso familiar}

Los niveles de persistencia son similares para todos los quintiles y descienden a medida que transcurren los semestres, desde alrededor de un 100\% en el primer semestre cursado, a cerca de un $80 \%$ en el séptimo semestre cursado. Si bien todos los quintiles tienen niveles 
de persistencia similares, el quinto quintil muestra un desempeño levemente más alto que el resto. Las diferencias son estadísticamente significativas solo en algunos semestres y cohortes, a favor del quinto quintil (Chi Cuadrado, 95\% de confianza). Para mayores detalles, ver Tabla 4.

Tabla 4: Porcentaje de alumnos persistentes según quintil de ingreso UC y año de admisión

\begin{tabular}{c|c|c|c|c|c|c|c|c|c|c}
\hline \multirow{2}{*}{ Quintil } & \multicolumn{5}{|c|}{2007} & \multicolumn{4}{|c|}{2008} & \multicolumn{2}{|c|}{2009} & 2010 \\
\cline { 2 - 11 } & $1 \mathrm{~s}$ & $3 \mathrm{~s}$ & $5 \mathrm{~s}$ & $7 \mathrm{~s}$ & $1 \mathrm{~s}$ & $3 \mathrm{~s}$ & $5 \mathrm{~s}$ & $1 \mathrm{~s}$ & $3 \mathrm{~s}$ & $1 \mathrm{~s}$ \\
\hline 1 & 97,8 & 94,4 & 87,8 & 83,3 & 98,0 & 87,3 & 82,9 & 98,3 & 91,3 & 99,4 \\
\hline 2 & 98,3 & 88,1 & 80,9 & $75,7 *$ & 97,0 & 88,7 & 83,5 & 97,5 & 90,8 & 97,6 \\
\hline 3 & 98,7 & 91,0 & 86,4 & 82,1 & 99,1 & 93,6 & 85,8 & 96,4 & $88,1 *$ & 98,8 \\
\hline 4 & 98,0 & 92,5 & 86,5 & 83,5 & 98,4 & 92,8 & 86,2 & 99,2 & 92,8 & 98,5 \\
\hline 5 & 98,4 & 91,6 & 87,0 & $84,3 *$ & 97,8 & 90,8 & 85,6 & 98,6 & $93,0 *$ & 98,4 \\
\hline Total & 98,3 & 91,5 & 86,5 & 83,4 & 97,9 & 90,8 & 85,3 & 98,4 & 92,3 & 98,4 \\
\hline$N^{0}$ total & 3.367 & 3.133 & 2.962 & 2.855 & 3.307 & 3.067 & 2.882 & 3.500 & 3.285 & 3.463 \\
\hline
\end{tabular}

Fuente: Elaboración propia. Información de la Dirección de Servicios y Registros Docentes, Vicerrectoría Académica UC. Alumnos matriculados, ingreso vía admisión ordinaria, en el primer semestre de cada año. Incluye campus Villarrica. Se excluye a los alumnos del Programa de Formación Pedagógica para Licenciados.

*: Grupos con diferencias estadísticamente significativas, con un $95 \%$ de confianza, siempre a favor del quinto quintil.

\section{Resumen y discusión de los resultados}

A continuación se presenta un resumen de los principales resultados de la investigación:

- En la mayoría de los semestres estudiados, los alumnos provenientes de colegios particulares pagados presentan promedios ponderados acumulados levemente más altos (alrededor de dos décimas) que sus pares que cursaron estudios en establecimientos particulares subvencionados y municipales. 
- En la comparación por quintil de ingreso, la diferencia más importante se observa para los alumnos provenientes del quinto quintil quienes presentaron, en algunas cohortes y periodos, promedios de notas levemente superiores a sus pares del segundo y cuarto quintil. Al igual que en el caso del análisis por dependencia, se trata de diferencias pequeñas que bordean las dos décimas de punto.

- Los resultados de persistencia también indican un mejor desempeño para los alumnos provenientes de colegios particulares pagados, de alrededor de tres puntos porcentuales, siendo estas diferencias estadísticamente significativas solo en algunos semestres estudiados respecto de los alumnos de colegios municipales.

- En relación con los resultados por quintil de ingreso, las tasas de persistencia son similares entre los distintos grupos, obteniendo los alumnos pertenecientes al quinto quintil tasas de persistencia levemente superiores en algunos períodos.

Si bien investigaciones anteriores, tanto en Chile como en Estados Unidos, han indicado que los estudiantes de menor nivel socioeconómico (es decir, con padres con menor educación o de menores ingresos) obtienen en general un peor desempeño que sus pares de nivel socioeconómico alto en términos de persistencia, la evidencia no es concluyente en términos de calificaciones una vez que se consideran las notas de educación secundaria y el puntaje en pruebas estandarizadas (Rothstein, 2004). Aún más, existen antecedentes que muestran que esa relación es pequeña y de limitada relevancia práctica cuando se compara el desempeño de alumnos que asisten a la misma institución o carrera, los que han sido sometidos a procesos de admisión exigentes y que, se puede suponer, cuentan con habilidades académicas similares (Geiser y Santelices, 2007).

A partir del análisis realizado en esta investigación, no es posible observar diferencias de relevancia práctica entre el desempeño académico (tanto notas como persistencia en la universidad) y nivel socioeconómico para los alumnos de la UC de las cohortes estudiadas. Más bien lo que se observa es un desempeño similar entre estudiantes de los distintos quintiles y dependencias educativas. Esto está en la 
línea de lo encontrado por Manzi (2006), Santelices (2007) y Geiser y Santelices (2007), quienes observan una asociación pequeña o nula entre antecedentes socioeconómicos (educación de los padres) y el rendimiento académico de los estudiantes en la educación superior. Una de las razones que podría estar explicando esta situación es el buen rendimiento previo de los alumnos que ingresan a la UC. Este alto rendimiento en términos de puntajes PSU y desempeño académico en la educación secundaria podría estar provocando que la relación entre la educación de los padres y el desempeño educativo de los hijos se vea atenuada. Relacionado con lo anterior, es importante considerar el perfil de los alumnos de bajo nivel socioeconómico que asiste a la UC: los estudiantes provenientes de colegios municipales "emblemáticos" representaron alrededor de la mitad de la matrícula de alumnos de establecimientos de dependencia municipal en la universidad ${ }^{13}$.

Si bien esta investigación se centra en las características de los estudiantes, en particular en su nivel socioeconómico, es factible que también existan características propias de la institución, tanto al interior de cada uno de los programas como a nivel institucional, que influyan en los resultados académicos de los alumnos. A nivel institucional, y como destaca el estudio de Microdatos (2008), a partir de mediados del año 2000 la UC introdujo una importante estrategia de apoyo a sus estudiantes, el CARA: Centro de Apoyo al Rendimiento Académico y Exploración Vocacional para los alumnos de la UC, que comenzó a ofrecer tutorías en el año 2005 y en 2007 se presentaba como un centro consolidado. Además de esta iniciativa, desde $2008^{14}$ existe dentro de la universidad la opción de cambiarse de carrera para los estudiantes que enfrentan dudas vocacionales, siempre y cuando su puntaje ponderado de admisión les hubiese alcanzado para matricularse en dicha carrera. Ambos aspectos, orientados a mejorar la experiencia universitaria de los jóvenes, podrían estar influyendo en los buenos resultados académicos obtenidos por los estudiantes

13 Un 57,2\% de los alumnos ingresados entre los años 2007 a 2010 a la UC desde colegios municipales egresó de los siguientes establecimientos: Instituto Nacional José Miguel Carrera, Liceo Carmela Carvajal de Prat, Liceo $N^{\circ} 1$ de Niñas, Liceo $N^{o} 7$ de Niñas de Providencia, Liceo José Victorino Lastarria, Liceo de Aplicación y Liceo Manuel Barros Borgoño (Fuente: Análisis propio sobre la base de información de DSRD).

14 Aunque desde 2006 como experiencia piloto. 
de la UC, aunque un análisis específico del impacto de este tipo de iniciativas sobre los indicadores académicos escapa a los límites de esta investigación.

Entre las principales proyecciones y limitaciones del estudio se puede mencionar que la capacidad de generalizar los resultados se ve limitada por las características propias de la UC, específicamente, por la particularidad de sus estudiantes, de sus programas académicos y del apoyo estudiantil que ofrece. Sin embargo, se considera que los resultados de esta investigación, si bien no son generalizables, pueden ser relevantes para otras instituciones de educación superior.

En relación con la metodología, una de las limitaciones del estudio es que los indicadores académicos fueron tratados de manera agregada, sin distinguir por disciplina o carrera. Estos aspectos pueden incidir en las medidas de rendimiento académico de los alumnos, en cuanto existen niveles de exigencia disímiles y escalas de calificaciones distintas entre carreras. Sin embargo, un análisis en términos de programa académico habría significado menores tamaños de muestra, dificultando el análisis inferencial.

Junto con evaluar la factibilidad de utilizar medidas de desempeño académico a nivel de disciplinas específicas, en futuros estudios podrían implementarse análisis multivariados, que permitan controlar diversas variables relevantes en el desempeño académico según la literatura, además de complementarse la información con otro tipo de indicadores académicos, como por ejemplo, la tasa de aprobación de cursos. Además, si bien este estudio siguió a los estudiantes durante varios periodos académicos, no fue posible hacer un seguimiento de cohortes hasta la graduación o titulación. Un seguimiento más a largo plazo de las carreras educativas de los estudiantes podría ser incorporado en estudios posteriores.

Pese a las limitaciones mencionadas con anterioridad, es importante destacar el principal hallazgo de este estudio: dentro de la UC, el desempeño académico de los alumnos de menores ingresos y provenientes de colegios municipales y particulares subvencionados es estadísticamente similar al de los alumnos de mayor ingreso y 
provenientes de colegios particulares pagados. De esta forma, este estudio entrega argumentos a favor de iniciativas para incorporar a alumnos de los primeros dos quintiles y que cursaron su enseñanza media en establecimientos municipales y particulares subvencionados, manteniendo la excelencia académica, como el programa de Talento e Inclusión UC. Sin embargo, es necesario tener en cuenta que cambios de mayor envergadura en los criterios de admisión a la universidad podrían tener efectos en la composición del cuerpo de estudiantes, ante lo cual los resultados académicos obtenidos por los distintos grupos podrían sufrir variaciones respecto de lo reportado en este estudio.

Por último y pese a lo anteriormente indicado se espera que esta investigación contribuya a la discusión tanto a nivel nacional como institucional respecto del acceso a la educación superior y el éxito académico de alumnos de bajo nivel socioeconómico, en especial en las universidades.

\section{Referencias}

Acuña, C., Makovec, M., \& Mizala, A. (2010). Access to higher education and dropouts: evidence from a cohort of Chilean secondary school leavers. Programa y Trabajos, Congreso Interdisciplinario de Investigación en Educación (CIIE). Recuperado el día 25 de octubre de 2011 desde http://www.ciie2010.cl/docs/doc/sesiones/183_MMakovec_Acceso_a_ ed_sup.pdf

Allen, J., Robbins, S., Casillas, A. \& Oh, I-S. (2008). Third-year college retention and transfer: effects of academic performance, motivation, and social connectedness. Research on Higher Education, 49, 647-664.

Barrios, M.A., Meneses, F. \& Paredes, R. (2011). Financial aid and university attrition in Chile. Manuscrito no publicado.

Bernstein, B. (1989). Clases, códigos y control. Estudios teóricos para una sociología del lenguaje. Madrid: Akal.

Bettinger, E. (2010). Need-based aid and student outcomes: the effects of the Ohio College Opportunity Grant. Manuscrito no publicado.

Bourdieu, P. \& Passeron, J.C. (2001). La reproducción: elementos para una teoría del sistema de enseñanza. Madrid: Popular. 
Canales, A. y De los Ríos, D. (2007). Factores explicativos de la deserción universitaria. Revista Calidad en la Educación, 26, 173-201.

Castillo, J. y Cabezas, G. (2010). Caracterización de jóvenes primera generación en educación superior. Nuevas trayectorias hacia la equidad educativa. Revista Calidad en la Educación, 32, 44-76.

Centro de Estudios Mineduc. (2012). Deserción en la educación superior en Chile. Centro de Estudios Mineduc, Serie Evidencias, Año 1, No 9. Recuperado el día 8 de noviembre de 2012 desde http://centroestudios. mineduc.cl/tp_enlaces/portales/tp5996f8b7cm96/uploadImg/File/ AlN9_Desercion.pdf

Chen, R. \& DesJardins, S. (2008). Exploring the effects of financial aid on the gap in student dropout risks by income level. Research on Higher Education, 49, 1-18.

(2010). Investigating the impact of financial aid on student dropout risks: racial and ethnic differences. The Journal of Higher Education, 81(2), 179-208.

Contreras, D., Gallegos, S. y Meneses, F. (2009). Determinantes de desempeño universitario: ¿Importa la habilidad relativa? Análisis exante y expost de una politica pública. Santiago: Consejo Nacional de Educación.

Dynarski, S. (2004). The new merit aid. En Hoxby, C. (Ed.) College Choices: The economics of where to go, when to go, and how to pay for it (pp. 63-97). Chicago: University of Chicago Press, National Bureau of Economic Research.

Geiser, S. \& Studley, R. (2002). UC and the SAT: predictive validity and differential impact of the SAT I and SAT II at the University of California. Educational Assessment, 8(1), 1-26.

Geiser, S. \& Santelices, M. V. (2006). The role of advanced placement and honors courses in college admissions. En Gandara, P., Orfield, G. \& Horn, C. L. (Eds.) Expanding opportunity in higher education: leveraging promise (pp. 75-114). Nueva York: State University of New York Press.

(2007). Validity of high school grades in predicting student success beyond the freshman year: high-school record vs. standardized tests as indicators of four-year college outcomes. Center for Studies on Higher Education, University of California, Berkeley, Research \& Occasional Paper Series, CSHE.6.07. Recuperado el día 25 de octubre de 2011 desde http://cshe.berkeley.edu/publications/ docs/ROPS.GEISER._SAT_6.12.07.pdf

Gil, F. y Del Canto, C. (2012). El caso del programa propedéutico en la Universidad de Santiago. Pensamiento educativo, 49(2), 65-83. 
González, L., Uribe, D. y González, S. (2005). Estudio sobre la repitencia y deserción en la educación superior chilena. Instituto Internacional para la Educación Superior en América Latina y el Caribe, Unesco. Recuperado el día 27 de octubre de 2011 desde http://unesdoc.unesco. org/images/0014/001400/140087s.pdf

Koljatic, M., \& Silva, M. (2012). Opening a side-gate: engaging the excluded in Chilean higher education through test-blind admission. Studies in Higher Education, 38(10), 1427-1441.

Kuh, G., Kinzie, J. Buckley, J. Bridges, B. \& Hayek, J. (2006). What matters to student success: a review of the literature. Commissioned report for the National Symposium on Postsecondary Student Success: Spearheading a Dialog on Student Success. Washington, DC: National Postsecondary Education Cooperative (NPEC).

Leyton, D., Vásquez, A. y Fuenzalida, V. (2012). La experiencia de estudiantes de contextos vulnerables en diferentes instituciones de educación superior (IESU): resultados de una investigación. Revista Calidad en la Educación, 37, 62-97.

Manzi, J. (2006). El acceso segmentado a la educación superior en Chile. En Fundación Equitas (Ed.), Caminos para la inclusión en la educación superior (pp. 187-204). Santiago: Fundación Equitas.

Manzi, J., Bravo, D., Del Pino, G., Donoso, G., Martínez, M. y Pizarro, R. (2006). Estudio acerca de la validez predictiva de los factores de selección a las universidades del Consejo de Rectores. Santiago: Comité Técnico Asesor de la PSU.

Meneses, F. y Toro, J. (2012). Predicción de notas en Derecho de la Universidad de Chile: ¿sirve el ranking? Revista ISEES, 10, 43-58.

Microdatos. (2008). Informe final estudio sobre las causas de la deserción universitaria. Santiago: Microdatos, Universidad de Chile.

Ministerio de Desarrollo Social. (2013). Estadísticas Educación Encuesta CASEN: Cobertura de educación superior por quintil de ingreso autónomo nacional según sexo. Observatorio Social, Ministerio de Desarrollo Social, Gobierno de Chile. Recuperado el día 10 de julio de 2013 desde http://observatorio.ministeriodesarrollosocial.gob.cl/casen/ casen-documentos.php?c=87\&a=2011

Organisation for Economic Co-operation and Development. (2009). La educación superior en Chile. Revisión de políticas nacionales de educación. Santiago: OECD/Ministerio de Educación de Chile.

Oswald, F., Schmitt, N., Kim, B., Ramsay, L. \& Gillespie, M. (2004). Developing a biodata measure and situational judgment inventory as 
predictors of college student performance. Journal of Applied Psychology, 89(2), 187-20.

Pascarella, E. T. \& Terenzini, P. T. (2005). How college affects students: a third decade of research. San Francisco: Jossey-Bass.

Rothstein, J. M. (2004). College performance predictions and the SAT. Journal of Econometrics, 121, 297-317.

Santelices, M. V. (2007). Desempeño académico de los alumnos admitidos a la Pontificia Universidad Católica de Chile. Manuscrito no publicado.

Santelices, M.V., Catalán, X., Horn, C. y Kruger, D. (2013). Determinantes de deserción en la Educación Superior Chilena, con énfasis en efecto de becas y créditos. Informe Final. Centro de estudios Mineduc, Concursos anteriores Fonide. Recuperado el día 15 de julio de 2013 desde http:// sgdce.mineduc.cl/descargar.php?id_doc=201304301626100

Servicio de Información de Educación Superior, SIES. (2008). Informe sobre retención de primer año de las carreras cohorte de ingreso 2007. Servicio de Información de la Educación Superior (SIES), reportes anuales: retención de pregrado. Recuperado el día 16 de agosto de 2012 desde http://www.mifuturo.cl/images/Informes_sies/Retencion/retencion_ pregrado_cohorte_2007_sies2008.pdf

(2009). Informe sobre retención de primer año de las carreras cohorte de ingreso 2008. Servicio de Información de Educación Superior (SIES). Reportes anuales: retención de pregrado. Recuperado el día 16 de agosto de 2012 desde http://www.mifuturo.cl/images/Informes_sies/ Retencion/retencion_pregrado_cohorte_2008_sies2009.pdf

(2010). Informe sobre retención de primer año de las carreras: cohorte de ingreso 2009 (Proceso SIES 2010). Servicio de Información de Educación Superior (SIES), reportes anuales: retención de pregrado. Recuperado el día 16 de agosto de 2012 desde http://www.mifuturo.cl/ images/Informes_sies/Retencion/retencion_pregrado_cohorte_2009_ sies2010.pdf

(2013). Listado de instituciones de educación superior vigentes. Servicio de Información de Educación Superior (SIES). Listado de instituciones. Recuperado el día 27 de agosto de 2013 desde http:// www.mifuturo.cl/images/Instituciones/instituciones_vigentes_ agosto_2013.xls

St. John, E., Hu, S. \& Fisher, A. (2011). Breaking through the access barrier: how academic capital formation can improve policy in higher education. Nueva York: Routledge. 
Stratton, L., Otoole, D, \& Wetzel, J. (2008). A multinomial logit model of college stopout and dropout behavior. Economics of Education Review, 27(3), 319-331.

Tinto, V. (2004). Student retention and graduation: facing the truth, living with the consequences. The Pell Institute, Occasional Paper $\mathrm{N}^{\circ} 1$, Pell Institute for the Study of Opportunity in Higher Education. Recuperado el día 5 de julio de 2012 desde http://www.pellinstitute.org/downloads/ publications-Student_Retention_and_Graduation_July_2004.pdf

Zwick, R. (2002). Fair game? The use of standardized admissions tests in higher education. Nueva York y Londres: Routledge Falmer.

Recibido: 02/09/2013

Aceptado: 20/05/2014 


\section{Anexo 1}

\section{Distribución por dependencia educativa del establecimiento de egreso y por quintil de ingreso familiar}

Figura 1: Distribución (\%) según dependencia educacional del colegio de egreso de enseñanza media en la UC de los alumnos matriculados en el período 1991 - 2011

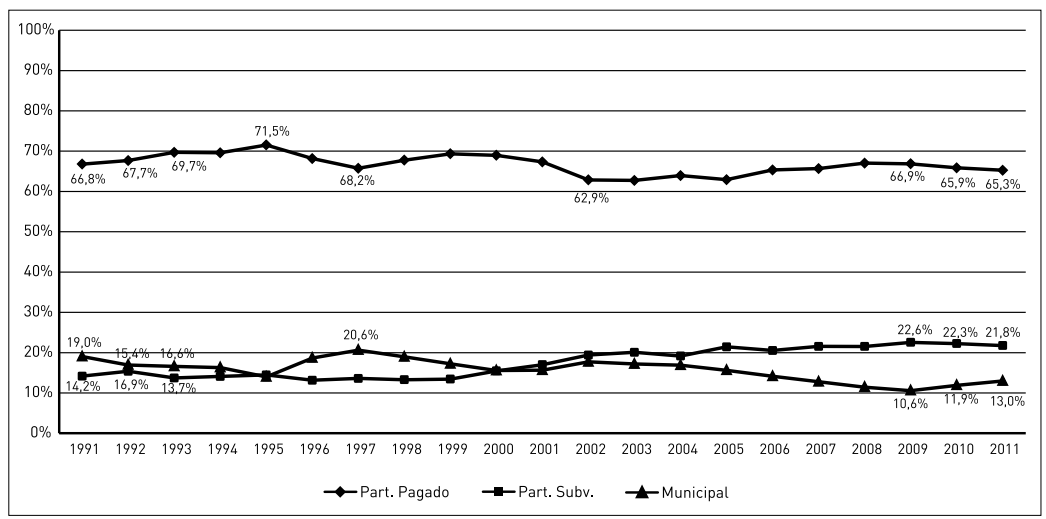

Fuente: Elaboración propia sobre la base de información de la Dirección de Servicios y Registros Docentes, Vicerrectoría Académica UC. Alumnos matriculados vía admisión ordinaria. No incluye Campus Villarrica.

Tabla 1: Distribución por quintil de ingreso según año de admisión

\begin{tabular}{c|c|c|c|c|c|c|c|c|c|c}
\hline \multirow{2}{*}{ Quintil de Ingreso } & \multicolumn{10}{|c|}{ Año } \\
\cline { 2 - 11 } & \multicolumn{2}{|c|}{2007} & \multicolumn{2}{|c|}{2008} & \multicolumn{2}{|c|}{2009} & \multicolumn{2}{c|}{2010} & \multicolumn{2}{|c}{2011} \\
\cline { 2 - 11 } & $n$ & $\%$ & $n$ & $\%$ & $n$ & $\%$ & $n$ & $\%$ & $n$ & $\%$ \\
\hline 1 & 90 & $2,6 \%$ & 260 & $7,5 \%$ & 121 & $3,2 \%$ & 158 & $4,2 \%$ & 130 & $3,5 \%$ \\
\hline 2 & 238 & $6,8 \%$ & 234 & $6,7 \%$ & 294 & $7,8 \%$ & 310 & $8,2 \%$ & 305 & $8,1 \%$ \\
\hline 3 & 304 & $8,6 \%$ & 229 & $6,6 \%$ & 286 & $7,6 \%$ & 267 & $7,1 \%$ & 256 & $6,8 \%$ \\
\hline 4 & 409 & $11,6 \%$ & 330 & $9,5 \%$ & 386 & $10,2 \%$ & 410 & $10,9 \%$ & 358 & $9,5 \%$ \\
\hline 5 & 2.484 & $70,4 \%$ & 2.438 & $69,7 \%$ & 2.686 & $71,2 \%$ & 2.620 & $69,6 \%$ & 2.721 & $72,1 \%$ \\
\hline Total & 3.525 & $100 \%$ & 3.491 & $100 \%$ & 3.773 & $100 \%$ & 3.765 & $100 \%$ & 3.770 & $100 \%$ \\
\hline
\end{tabular}

Fuente: Elaboración propia sobre la base de información del Departamento de Asistencia Socioeconómica. Alumnos matriculados, ingreso vía admisión ordinaria. Incluye campus Villarrica.

*: A los alumnos que en los datos entregados figuraban como pertenecientes al quinto quintil de ingreso se sumaron aquellos alumnos que, sobre la base de los datos, aparecen sin información sobre esta variable. Ya que este dato existe para todos aquellos que reciben beneficios socioeconómicos, se maneja la hipótesis de que quienes no los reciben y no cuentan con información sobre quintil debieran pertenecer, en su mayoría, al quinto quintil de ingreso. 


\begin{tabular}{|c|c|c|c|c|c|c|c|c|c|}
\hline \multirow{18}{*}{ 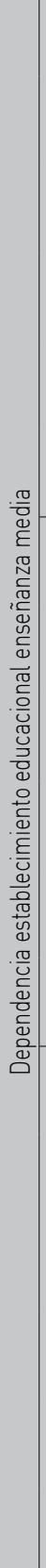 } & \multirow{6}{*}{ 客 } & \multirow{2}{*}{ 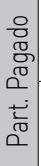 } & \multirow{2}{*}{\begin{tabular}{|l} 
。 \\
$c$
\end{tabular}} & $\widetilde{\sigma}$ & - & $\sim$ & $\stackrel{\circ}{\mathcal{F}^{-}}$ & $\stackrel{\mathfrak{N}}{\mathrm{N}}$ & ᄋ̊ \\
\hline & & & & $\checkmark$ & $\underset{\sim}{\beth}$ & 요 & 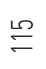 & 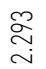 & $\stackrel{\circ}{\stackrel{\circ}{\sim}}$ \\
\hline & & $\begin{array}{l}0 \\
\stackrel{0}{0} \\
\check{0}\end{array}$ & $\circ^{\circ}$ & $\stackrel{\infty}{\sim}$ & $\begin{array}{l}\sigma^{-} \\
-\end{array}$ & $\infty^{-}$ & $\approx$ & $\begin{array}{l}\stackrel{L}{-} \\
\stackrel{\text { m}}{ }\end{array}$ & ㅇㅇㅇ \\
\hline & & 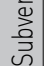 & $c$ & $\stackrel{\infty}{\infty}$ & $\stackrel{\Xi}{\ni}$ & $\stackrel{\Re}{\longrightarrow}$ & $\stackrel{\varrho}{\varrho}$ & ్ా & $\overleftarrow{\infty}$ \\
\hline & & $\vec{\pi}$ & $\circ^{\circ}$ & $\simeq$ & $\stackrel{\infty}{\infty}$ & $\stackrel{\circ}{\check{E}}$ & $\bar{\sigma}$ & $\stackrel{\circ}{\check{\sim}}$ & ઠे \\
\hline & & $\stackrel{\text { క̦ }}{\Sigma}$ & $\subset$ & $\stackrel{\gamma}{\gamma}$ & $\cong$ & $\approx$ & $\stackrel{\infty}{\simeq}$ & $\infty$ & 字 \\
\hline & & $\begin{array}{l}0 \\
8 \\
0 \\
0\end{array}$ & $\alpha^{\circ}$ & $\bar{\sigma}$ & $\stackrel{\circ}{\circ}$ & $\sim$ & مـ & $\frac{+}{\sigma}$ & 융 \\
\hline & & 它 & $z$ & $\approx$ & $\bar{\sim}$ & io & $\stackrel{\llcorner 0}{=}$ & $\stackrel{\stackrel{\Omega}{O}}{\underset{\sim}{\sim}}$ & ָे \\
\hline & $\infty$ & $\begin{array}{l}0 \\
0 \\
0 \\
.0 \\
.0\end{array}$ & 。 & $\stackrel{\sim}{\infty}$ & $\mathfrak{m}_{0}^{-}$ & $\underset{\mathcal{I}}{\stackrel{\sim}{ \pm}}$ & $\stackrel{\infty}{\infty^{-}}$ & $\widehat{\widehat{్}}$ & ஜे \\
\hline & & 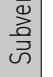 & $\subset$ & ले & $\stackrel{\stackrel{2}{\simeq}}{\simeq}$ & gे & $\stackrel{\Im}{\Xi}$ & 足 & $\stackrel{2}{2}$ \\
\hline & & 즘 & $\circ^{\circ}$ & $\begin{array}{l}\infty \\
\stackrel{\sim}{\sim}\end{array}$ & 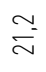 & $\stackrel{\infty}{=}$ & $\stackrel{\llcorner}{\stackrel{2}{=}}$ & $\begin{array}{l}\infty \\
\infty^{-}\end{array}$ & 응 \\
\hline & & $\sum^{\frac{5}{2}}$ & $\subset$ & 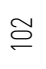 & 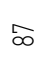 & $\mathbb{2}$ & $\simeq$ & $\approx$ & $\bar{\ddagger}$ \\
\hline & & $\begin{array}{l}8 \\
8 \\
0 \\
0 \\
0\end{array}$ & $\circ^{\circ}$ & $\widetilde{\sigma}$ & J & $\sim$ & ما & $\stackrel{\llcorner}{\sim}$ & 으 \\
\hline & & 䒕 & $c$ & مص & $\infty$ & 우 & 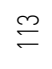 & 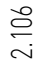 & 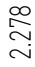 \\
\hline & & $\begin{array}{l}0 \\
\stackrel{0}{0} \\
.0\end{array}$ & ঃ & $\mathrm{L}^{t}$ & $\approx$ & $\begin{array}{l}\stackrel{\sigma}{\circ} \\
\stackrel{0}{\infty}\end{array}$ & $\widetilde{\approx}$ & 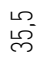 & 으 \\
\hline & & $\begin{array}{l}\text { 今a } \\
\text { âे } \\
\omega\end{array}$ & $\subset$ & $\underset{F}{ }$ & $\widetilde{\varpi}$ & 齐 & $\stackrel{\varnothing}{\varnothing}$ & $\stackrel{\Xi}{\beth}$ & 尺 \\
\hline & & $\widetilde{\widetilde{\sigma}}$ & $0^{\circ}$ & $\approx$ & $\overline{\bar{\sim}}$ & $\stackrel{\sim}{\sim}$ & $\sqrt{\sim}$ & $\stackrel{2}{\stackrel{\sim}{\sim}}$ & 으 \\
\hline & & $\sum^{\frac{5}{2}}$ & $c$ & $\Im$ & 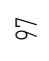 & $\Xi$ & $\stackrel{\llcorner}{=}$ & 丈 & 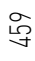 \\
\hline & 害 & & & - & $\sim$ & $m$ & $\checkmark$ & م & 䨐 \\
\hline
\end{tabular}




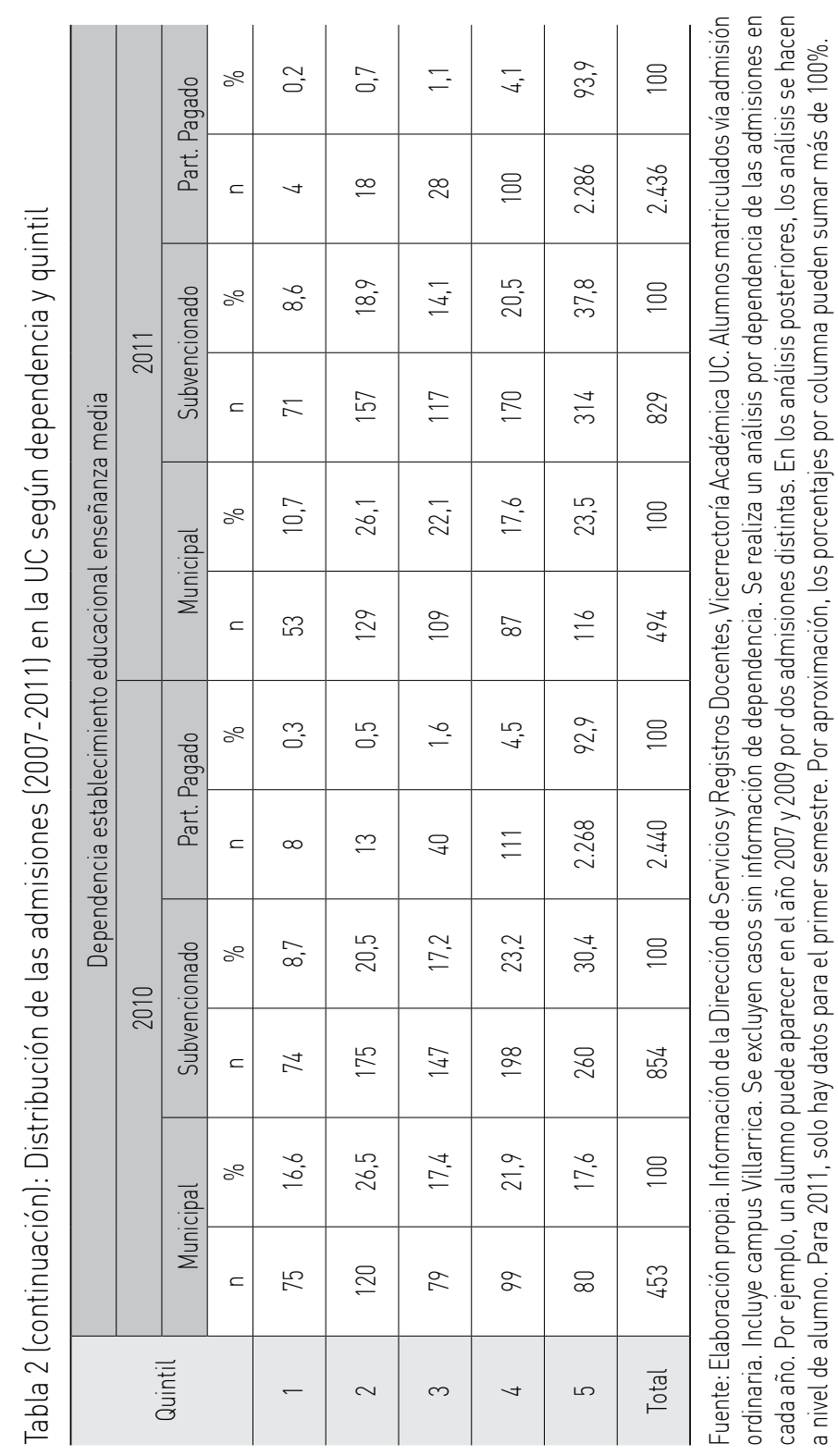




\section{Anexo 2}

\section{Diferencias entre alumnos ingresados vía admisión ordinaria y especial}

Las diferencias entre los alumnos ingresados vía admisión especial y vía admisión ordinaria a la UC son relevantes considerando que en los últimos tres años los alumnos ingresados vía admisión especial han representado aproximadamente el 15\% del total de los alumnos matriculados (ver Tabla 1).

Tabla 1: Distribución de las admisiones (2007-2011) en la UC según vía de ingreso

\begin{tabular}{l|c|c|c|c|c|c|c|c|c|c}
\hline \multirow{2}{*}{ Vía de admisión } & \multicolumn{2}{|c|}{2007} & \multicolumn{2}{c|}{2008} & \multicolumn{2}{c|}{2009} & \multicolumn{2}{|c|}{2010} & \multicolumn{2}{|c}{2011} \\
\cline { 2 - 11 } & $\mathrm{n}$ & $\%$ & $\mathrm{n}$ & $\%$ & $\mathrm{n}$ & $\%$ & $\mathrm{n}$ & $\%$ & $\mathrm{n}$ & $\%$ \\
\hline Admisión ordinaria & 3.524 & $84,6 \%$ & 3.491 & $83,9 \%$ & 3.773 & $84,7 \%$ & 3.765 & $82,6 \%$ & 3.770 & $83,8 \%$ \\
\hline Admisión especial & 640 & $15 \%$ & 672 & $16,1 \%$ & 680 & $15,3 \%$ & 793 & $17,4 \%$ & 726 & $16,2 \%$ \\
\hline Total & 4.164 & $100 \%$ & 4.163 & $100 \%$ & 4.453 & $100 \%$ & 4.558 & $100 \%$ & 4.496 & $100 \%$ \\
\hline
\end{tabular}

Fuente: Elaboración propia. Información de la Dirección de Servicios y Registros Docentes, Vicerrectoría Académica UC. Alumnos matriculados vía admisión ordinaria y especial. Incluye campus Villarrica. Se realiza un análisis por dependencia de las admisiones en cada año. Por ejemplo, un alumno puede aparecer en el año 2007 y 2009 por dos admisiones distintas. En los análisis posteriores, los análisis se hacen a nivel de alumno. Para 2011, solo hay datos para el primer semestre.

\section{a. Dependencia educativa}

Al comparar a los alumnos de admisión ordinaria y admisión especial según la dependencia educacional del colegio de enseñanza media, se observa que en el periodo estudiado, si bien en ambos grupos los alumnos provenientes de establecimiento particulares pagados fueron mayoría, tuvieron una menor representación en el grupo de alumnos que ingresó vía admisión especial (alrededor de 10 puntos porcentuales menos).

\section{b. Promedio ponderado acumulado}

Al comparar el PPA según vía de ingreso se observa que, en general, quienes ingresaron vía admisión especial obtuvieron promedios 
levemente más altos (entre una y dos décimas más) que quienes lo hicieron vía admisión ordinaria. Esto ocurrió para los alumnos provenientes de todas las dependencias educacionales, especialmente para la dependencia particular pagada, aunque solo en algunos casos las diferencias fueron estadísticamente significativas.

\section{c. Persistencia}

El porcentaje de alumnos persistentes fue levemente menor para quienes ingresaron vía admisión especial en los tres años estudiados y para los tres tipos de dependencia educativa (persistencia entre 65\% y $70 \%$ al séptimo semestre para los alumnos de ingreso vía admisión especial, versus porcentajes de entre $80 \%$ y $85 \%$ para ese mismo periodo en el caso de los alumnos de ingreso a través de admisión ordinaria). Esto refleja el mayor porcentaje del primer grupo (admisión especial) que egresó y/o se tituló (menos del 1\% para los alumnos de admisión ordinaria y entre $8 \%$ y $13 \%$ para los alumnos de admisión especial). Las diferencias fueron estadísticamente significativas para el séptimo semestre. 